\title{
Bioinformatic Analysis Identifies Biomarkers and Treatment Targets in Primary Sjögren's Syndrome Patients with Fatigue
}

\author{
Guangshu Chen $\mathbb{D}^{1},{ }^{1}$ Li Che $\mathbb{D},{ }^{2}$ Xingdong Cai $\mathbb{D}^{2},{ }^{2}$ Ping Zhu $\mathbb{D},{ }^{1}$ and Jianmin Ran $\mathbb{D}^{1}$ \\ ${ }^{1}$ Department of Endocrinology, Guangzhou Red Cross Hospital, Jinan University, Guangzhou 510220, China \\ ${ }^{2}$ Department of Pulmonary and Critical Care Medicine, The First Affiliated Hospital of Jinan University, Guangzhou 510630, China
}

Correspondence should be addressed to Jianmin Ran; ran_jianmin@ext.jnu.edu.cn

Received 3 November 2021; Accepted 23 December 2021; Published 15 January 2022

Academic Editor: Paul Harrison

Copyright (c) 2022 Guangshu Chen et al. This is an open access article distributed under the Creative Commons Attribution License, which permits unrestricted use, distribution, and reproduction in any medium, provided the original work is properly cited.

\begin{abstract}
We aim to identify the common genes, biological pathways, and treatment targets for primary Sjögren's syndrome patients with varying degrees of fatigue features. We select datasets about transcriptomic analyses of primary Sjögren's syndrome (pSS) patients with different degrees of fatigue features and normal controls in peripheral blood. We identify common differentially expressed genes (DEGs) to find shared pathways and treatment targets for pSS patients with fatigue and design a protein-protein interaction (PPI) network by some practical bioinformatic tools. And hub genes are detected based on the PPI network. We perform biological pathway analysis of common genes by Gene Ontology (GO) terms and Kyoto Encyclopedia of Genes and Genomes (KEGG) pathway. Lastly, potential treatment targets for pSS patients with fatigue are found by the Enrichr platform. We discovered that 27 DEGs are identified in pSS patients with fatigue features and the severe fatigued pSS-specific gene is RTP4. DEGs are mainly localized in the mitochondria, endosomes, endoplasmic reticulum, and cytoplasm and are involved in the biological process by which interferon acts on cells and cells defend themselves against viruses. Molecular functions mainly involve the process of RNA synthesis. The DEGs of pSS are involved in the signaling pathways of viruses such as hepatitis C, influenza A, measles, and EBV. Acetohexamide PC3 UP, suloctidil HL60 UP, prenylamine HL60 UP, and chlorophyllin CTD 00000324 are the four most polygenic drug molecules. PSS patients with fatigue features have specific gene regulation, and chlorophyllin may alleviate fatigue symptoms in pSS patients.
\end{abstract}

\section{Introduction}

Primary Sjögren's syndrome (pSS) is an all-body autoimmune disease that mainly affects middle-aged women [1]. The main clinical feature of the disease is dryness of the mouth and eyes, and the pathophysiology is characterized by focal lymphocyte infiltration in exocrine glands $[2,3]$. Fatigue is commonly seen in pSS patients as an extraglandular manifestation and closely links with poor life quality [4-6]. Fatigue affects approximately $70 \%$ of pSS patients $[7,8]$. Normally, fatigue and depression are considered manifestations of psychological disorders and interact with physical pain and discomfort, which creates a vicious cycle. Fatigue in pSS is induced and regulated by genetic and molecular mechanisms, with the innate immune system playing an important role in the produc- tion of fatigue [9-11]. Although pSS always comes with fatigue, not all patients exhibit fatigue, which provides a good model for exploring the underlying biological mechanisms.

High-throughput methods play an increasingly essential role in biology spheres, and microarray data analysis highlights its advantage in large-scale analysis of gene expression among high-throughput applications [12,13]. Former studies $[14,15]$ have shown the high-throughput sequencing analysis result for pSS patients with fatigue features but do not offer further analysis based on varying degrees of fatigue. This study tries to present characteristic genes and biological pathways in pSS patients with manifestations of fatigue, as well as drugs of potential benefit.

The GSE66795 dataset from the GPL10558 platform on the GEO database is selected for gene expression of pSS with 
fatigue. The GSE66795 dataset was first identified for differentially expressed genes (DEGs) in pSS patients with different levels of fatigue, and based on the coexpressed genes, further analyses including Gene Ontology (GO) terms and Kyoto Encyclopedia of Genes and Genomes (KEGG) pathway are performed to understand the biological process. The top ten target genes from the protein-protein interaction (PPI) network will be obtained to identify potential drugs that may alleviate fatigue in pSS patients.

\section{Materials and Methods}

2.1. Dataset Collection. We search “Primary Sjögren's syndrome" and "fatigue" in the GEO database [16] and select the dataset (GSE66795) demonstrating gene expression in pSS patients with varying degrees of fatigue characteristics and normal controls. The GSE66795 dataset is extracted from the GPL10558 platform (Illumina HumanHT-12 V4.0 expression microbead chip) for RNA sequence analysis. The data of GSE66795 is obtained from the UK registry of primary Sjögren's syndrome. It includes whole genome microarray profiles of pSS patients with varying degrees of fatigue characteristics and normal controls in peripheral blood. One hundred and thirty-one patients with pSS are involved, including 21 patients with mild fatigue, 74 patients with moderate fatigue, 36 patients with severe fatigue, and 29 normal controls.

2.2. Differential Expression Analysis. Differential expression analysis is performed using the online analysis tool GEO2R; gene expression profiles of pSS patients with mild, moderate, and severe fatigue were compared with normal controls separately to identify DEGs. $P$ values and adjusted $P$ values are calculated using $t$-tests. Genes with the following criteria were retained for each sample: (1) $\log 2$-fold change $(\log 2 \mathrm{FC})$ absolute value greater than 1 and (2) adjusted $P$ value less than 0.05 . After identifying DEGs in pSS patients with varying degrees of fatigue, the online website (https://www.xiantao.love/gds) is used to plot a Venn diagram.

2.3. Gene Ontology and Pathway Discovery in Gene Set Enrichment Analysis. Gene set enrichment analysis is used to understand the general biological function and the chromosomal location of a gene [17]. For gene product annotation, the terms of Gene Ontology (GO) are used, including biological process (BP), molecular function (MF), and cellular component (CC) [18]. The Kyoto Encyclopedia of Genes and Genomes (KEGG) pathways are commonly used to describe metabolic pathways [19]. GO terms and KEGG pathways were gotten through the platform Enrichr (https://amp.pharm.mssm.edu/Enrichr/) based on the DEGs [20].

2.4. Protein-Protein Interaction (PPI) Network. The information generated from the PPI network improves the understanding of protein function [21]. PPI networks are made by STRING (https://string-db.org/) after inputting the common DEGs. We analyze PPIs through Cytoscape (https:// cytoscape.org/) to further present the network and identify target genes.

2.5. Transcription Factor- (TF-) Gene Interactions. We use NetworkAnalyst (https://www.networkanalyst.ca/) to identify interactions of TF-genes with DEGs [22]. NetworkAnalyst plays a comprehensive network platform for gene expression across a wide range of species and enables them to be subjected to a meta-analysis [23].

2.6. Identification of Potential Treatment Targets. Identification of drug molecules is a vital component of genomics research. We input the DEGs in the Drug Signature Database (DSigDB). Then, we get the designed drug molecules, which may have promising clinical application. DSigDB is obtained through the Enrichr (https://amp .pharm.mssm.edu/Enrichr/) platform. Enrichr is primarily used as an enrichment analysis platform, providing extensive visual details of the common functions of inputted genes [24].

\section{Results}

3.1. DEG Identifications. We use the GSE66795 dataset to identify the DEGs of pSS with fatigue. 37, 29, and 33 DEGs are obtained for pSS with mild, moderate, and severe fatigue, respectively. The collected DEGs are further compared by using the online website (https://www .xiantao.love/gds) for gathering common genes in pSS with varying degrees of fatigue. And 27 (OAS1, OAS2, GBP1, IRF7, EIF2AK2, IFIT2, USP18, SAMD9L, HES4, IFI44L, SERPING1, IFIT3, IFITM3, IFI6, XAF1, MX1, OASL, OTOF, HERC5, LY6E, EPSTI1, OAS3, ISG15, IFIT1, RSAD2, IFI44, and IFI27) common DEGs are identified. The specific genes to pSS with mild fatigue are DDX60, IFIH1, GBP5, LAP3, and TIMM10. The specific genes to pSS with moderate fatigue are HLA-DRB4 and HLADRB6, and that to pSS with severe fatigue is RTP4. The Venn diagram (Figure 1) shows that common DEGs accounted for $67.5 \%$ out of a total of 40 DEGs.

3.2. GO Terms and KEGG Pathways. We analyzed 27 common DEGs for both GO and KEGG pathways. Both of the results are taken from the top $10 \mathrm{GO}$ entries. GO terms in Table 1 suggest that DEGs are mainly localized in the mitochondria, endosomes, endoplasmic reticulum, and cytoplasm. They are involved in the biological processes of interferon action on cells and cellular defense against viruses. And the molecular functions are mainly engaged in the process of RNA synthesis. KEGG pathways in Table 2 suggest that the DEGs of pSS with fatigue are involved in the signaling pathways of viruses such as hepatitis C, influenza A, measles, and EBV. Both are seen in Figures 2(a) and 2(b).

3.3. Identification of Hub Genes by PPI Networks. We put common DEGs into the STRING website, and the files generated after analysis are further entered into Cytoscape software for visual analysis. PPI networks are designed to detect hub genes for identifying drug molecules for pSS with 


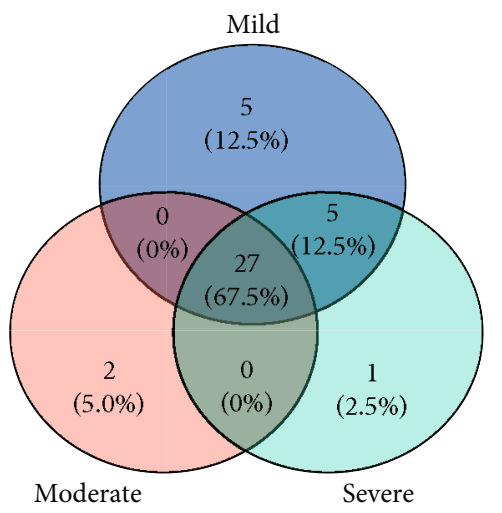

FIgURE 1: A Venn diagram represents common DEGs. Twentyseven genes are found common among the 40 DEGs of pSS patients with varying degrees of fatigue. The common DEGs accounted for $67.5 \%$ out of a total of 40 DEGs.

fatigue. PPI networks involve 24 nodes and 552 edges, which are shown in Figure 3(a). We present the top 20 genes in Figure 3(b) and Table 3.

3.4. TF-Gene Interactions. The interactions of TF and genes are shown in Figure 4. The network has 60 nodes and 108 edges. Sixteen TF-genes regulate IFIT1, and IFIT3 is handled by 14 TF-genes. The network involves 60 TF-genes. Figure 4 shows the network of TF-gene interactions.

3.5. Identification of Drug Candidates. We identify drug molecules for the top 10 hub genes on the Enrichr platform. We collect drug candidates judged on adjusted $P$ values. The analysis reveals that acetohexamide PC3 UP, suloctidil HL60 UP, prenylamine HL $60 \mathrm{UP}$, and chlorophyllin CTD 00000324 are the four most polygenic drug molecules that interact with genes. Figure 5 and Table 4 present the drug candidates in DSigDB.

\section{Discussion}

Fatigue is an annoying experience that means physical and mental tiredness [25]. Mengshoel et al. [26] reveal that most pSS patients literally suffer from fluctuating fatigue out of control regardless of their health condition. Fatigue has a significant influence on patients' daily life, and patients must adapt to their behavior and lives. Although the underlying mechanisms are still unclear, former studies take depression and pain as the prominent factors associated with fatigue [5, 27]. Currently, growing evidence suggests that fatigue has a molecular and genetic basis on its production and regulation. Therefore, most scholars view fatigue as a biological and brain phenomenon [9-11].

IL- $1 \beta$ tends to increase rapidly secreted from macrophages to activate the immune system when meeting tissue injury or infection. IL- $1 \beta$ plays its role by binding with the IL-1 receptor coming with the downstream of IL-1 response [28]. Then, immune and inflammation systems are activated, which induce the behavior of disease, with fatigue being involved as an important component [29]. All these inflammatory signaling pathways go on working and turn fatigue into a chronic state. In the brain, IL-1 $\beta$ signaling pathways may explain the ultimate pathway of fatigue [30, 31], and IL-1 blocker treatment may effectively release fatigue $[32,33]$. Thus, fatigue and other unpleasant mood in those patients with autoimmune disease not only should be understood by the unfortunate development of chronic illness but also may be related to some signaling pathways and activation of genes that regulate the mood in the cerebral system.

Genome-wide association analysis of pSS patients has been conducted, and a gene (RTP4) is identified as highly relevant. Similarly, we confirm that RTP4 is highly expressed in pSS patients with severe fatigue through bioinformatic analysis, suggesting that this gene is critical in the mechanisms of fatigue. RTP4 encodes a protein associated with the expression of opioid receptors on the cell surface. These receptors are also expressed in the lymph system and painregulated pathways in the brain [34]. However, the former study did not stratify pSS based on the degree of fatigue, and it is unclear which degree of fatigue expresses the RTP4 gene. Our study finds that pSS patients with severe fatigue specifically express the RTP4 gene, providing clues for further studies on the genomics of fatigue features in pSS patients.

OAS1, a coexpressed gene for pSS in our study, has been established in previous studies as a risk locus of pSS and impacts the flaw of virus clearance because of the altering response of IFN [35]. Our gene pathway analysis points out that DEGs for pSS with fatigue are mainly localized intracellularly and involved in signaling pathways of common viruses in the respiratory and digestive tracts, suggesting that pSS is a systemic disease with an uncertain etiology and that viral infection may be a predisposing factor.

Fatigue always accompanies pSS patients, but it is hard work to manage these bad feelings [36]. The clinical practice guidelines (CPG) committee emphasizes the many causes of fatigue in pSS; therefore, the comprehensive evaluation for diagnosis is essential. So far, the treatment for fatigue in pSS with solid recommendation is mere taking exercise, which is also practical in other autoimmune diseases [37]. In America, hydroxychloroquine (HCQ) is the most widely used drug therapy for pSS with fatigue, but the recommendation strength is not strong enough [34]. It is not recommended to release fatigue in pSS using dehydroepiandrosterone (DHEA) [34]. Both the tumor necrosis factor inhibitor is discouraged for the treatment of fatigue in pSS [38, 39]. Our bioinformatic study reveals that besides chloroquine and testosterone drugs that help improve fatigue, chlorophyllin, the sulphonylurea hypoglycaemic drug acetylhexane, and the antiallergic drug terfenadine may have improved fatigue in pSS. However, chloroquine and testosterone are not strongly recommended as we mentioned before. Acetohexamide has been discontinued in the American market due to its significant hypoglycaemic risk. Terfenadine is not suitable for long-term use since its 
TABle 1: Top 10 GO pathways and their corresponding $P$ values and genes.

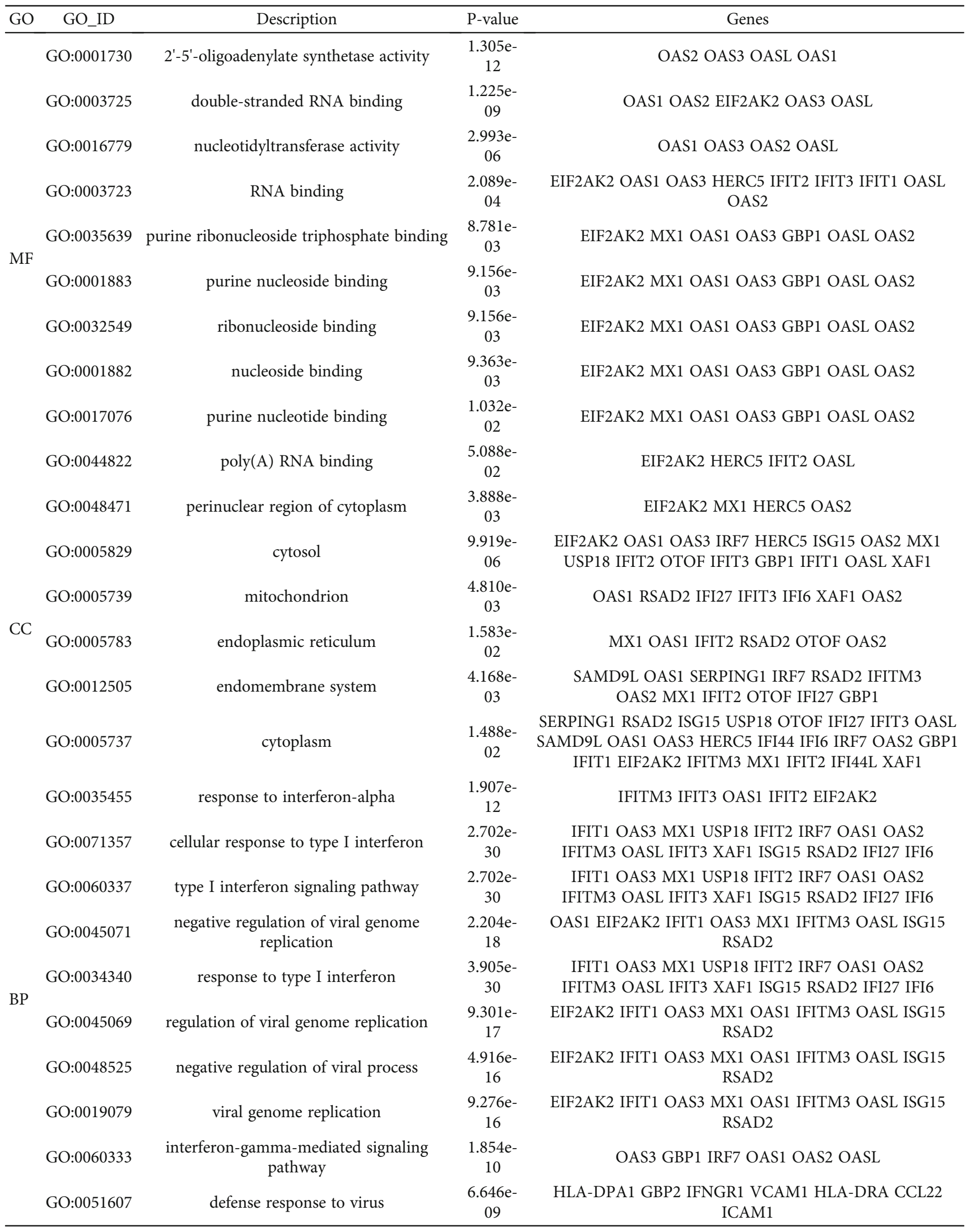


TABLE 2: Top 10 KEGG pathways and their corresponding $P$ values and genes.

\begin{tabular}{lccc}
\hline Pathway ID & Description & $P$ value & Genes \\
\hline hsa05160 & Hepatitis C & $2.02329 e-11$ & IFIT1 IRF7 MX1 OAS1 OAS2 OAS3 EIF2AK2 RSAD2 \\
hsa05164 & Influenza A & $2.60307 e-09$ & IRF7 MX1 OAS1 OAS2 OAS3 EIF2AK2 RSAD2 \\
hsa05162 & Measles & $3.6267 e-08$ & IRF7 MX1 OAS1 OAS2 OAS3 EIF2AK2 \\
hsa05169 & Epstein-Barr virus infection & $3.37146 e-07$ & IRF7 OAS1 OAS2 OAS3 EIF2AK2 ISG15 \\
hsa04621 & NOD-like receptor signaling pathway & $5.95267 e-06$ & GBP1 IRF7 OAS1 OAS2 OAS3 \\
hsa05165 & Human papillomavirus infection & 0.000110032 & MX1 EIF2AK2 OASL ISG15 HES4 \\
hsa05168 & Herpes simplex virus 1 infection & 0.000742976 & IRF7 OAS1 OAS2 OAS3 EIF2AK2 \\
hsa04622 & RIG-I-like receptor signaling pathway & 0.005431097 & IRF7 ISG15 \\
hsa05167 & Kaposi sarcoma-associated herpesvirus infection & 0.037274554 & IRF7 EIF2AK2 \\
hsa05203 & Viral carcinogenesis & 0.041244055 & IRF7 EIF2AK2
\end{tabular}

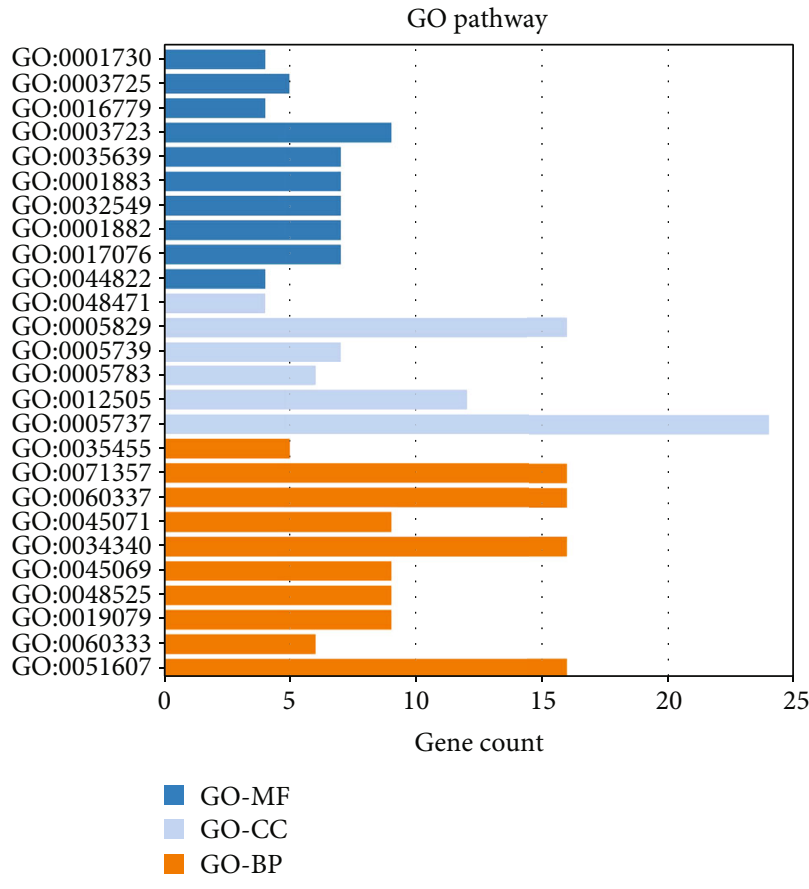

(a)

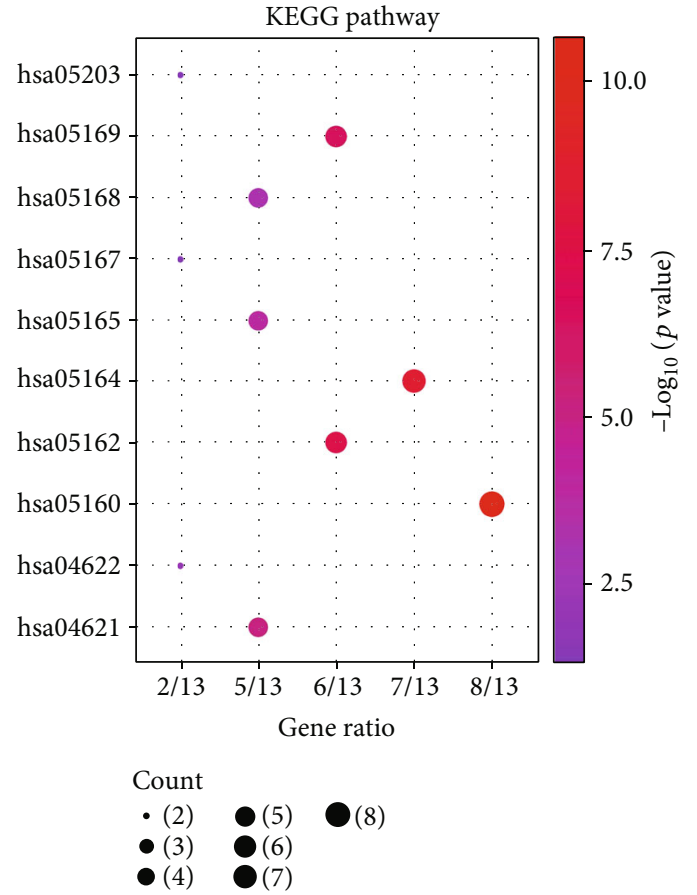

(b)

Figure 2: (a) The result of biological process-, molecular function-, and cellular component-associated GO terms. (b) The result of KEGG pathway analysis.

central depression as an antiallergy drug. And chlorophyllin appears to hold some promise for reducing fatigue in pSS.

Chlorophyll is an ingredient of the derifil drug which is available as an over-the-counter medicine [40]. And chlorophyllin, obtained by hydrolyzing chlorophyll to remove phytyl alcohol, is a water-soluble derivative. Chlorophyll has been shown to exert its anticancer properties by playing a role as an antioxidant [41], a CYP inhibitor [42], an apoptosis inducer [43], a phase II enzyme stimulator [44], and a carcinogen transport modulator [45]. Currently, COVID-19 has swept the world and may last for a long time because of its rapid mutation. Almost
$5,000,000$ people have died in this epidemic [46], and the reduction of lymphocytes in COVID-19 patients is considered an important risk factor for poor prognosis [47-49]. Recent studies suggest that the chlorophyll derivative sodium copper chlorophyllin (SCC) may improve survival in critically ill COVID-19 patients by increasing the total number of lymphocytes [50]. Increasing consumers choose dietary chlorophyll which is derived from SCC for diet supplements for the sake of keeping healthy [51, 52]. Dietary chlorophyll is safe and has been shown to have a higher absorption rate in the human body, which may trigger ionic compound chelation [53, 54]. Zeng et al. [55] cognize one functional food called barley grass 


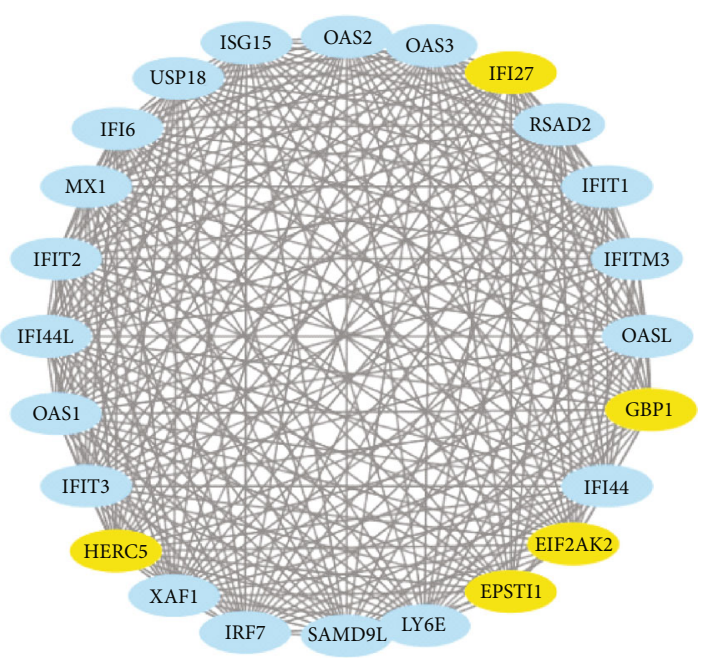

(a)

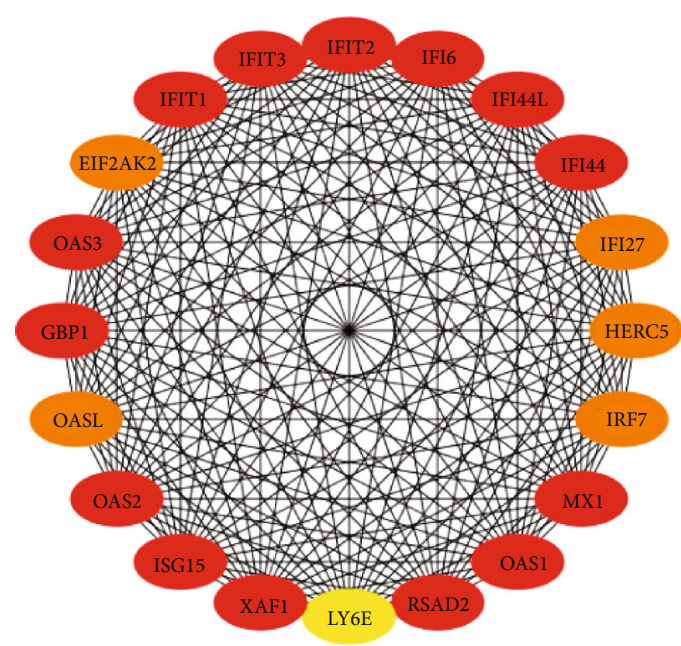

(b)

Figure 3: (a) Protein-protein interaction (PPI) network for DEGs of patients with varying degrees of fatigue. Nodes in blue color indicate common DEGs with the highest connection degree value, and edges specify the interconnection in the middle of two genes. The network involves 24 nodes and 552 edges. (b) The top 20 genes are detected from the PPI network of common DEGs. The nodes in red color represent 14 genes with the highest degree value which are MX1, IFIT1, ISG15, RSAD2, IFI44L, IFI44, IFIT3, OAS2, OAS1, IFI6, XAF1, IFIT2, GBP1, and OAS3. Based on the topological analysis, the degree value of these 14 genes is both 23.

TABLE 3: Topological result exploration for top 20 genes.

\begin{tabular}{|c|c|c|c|c|c|c|}
\hline Hub gene & Degree & Stress & Closeness & Betweenness & Eccentricity & Clustering coefficient \\
\hline MX1 & 23 & 20 & 23 & 1.09235 & 1 & 0.96047 \\
\hline IFIT1 & 23 & 20 & 23 & 1.09235 & 1 & 0.96047 \\
\hline ISG15 & 23 & 20 & 23 & 1.09235 & 1 & 0.96047 \\
\hline RSAD2 & 23 & 20 & 23 & 1.09235 & 1 & 0.96047 \\
\hline IFI44L & 23 & 20 & 23 & 1.09235 & 1 & 0.96047 \\
\hline IFI44 & 23 & 20 & 23 & 1.09235 & 1 & 0.96047 \\
\hline IFIT3 & 23 & 20 & 23 & 1.09235 & 1 & 0.96047 \\
\hline OAS2 & 23 & 20 & 23 & 1.09235 & 1 & 0.96047 \\
\hline OAS1 & 23 & 20 & 23 & 1.09235 & 1 & 0.96047 \\
\hline IFI6 & 23 & 20 & 23 & 1.09235 & 1 & 0.96047 \\
\hline XAF1 & 23 & 20 & 23 & 1.09235 & 1 & 0.96047 \\
\hline IFIT2 & 23 & 20 & 23 & 1.09235 & 1 & 0.96047 \\
\hline GBP1 & 23 & 20 & 23 & 1.09235 & 1 & 0.96047 \\
\hline OAS3 & 23 & 20 & 23 & 1.09235 & 1 & 0.96047 \\
\hline HERC5 & 22 & 14 & 22.5 & 0.75624 & 0.5 & 0.9697 \\
\hline IRF7 & 22 & 10 & 22.5 & 0.51637 & 0.5 & 0.97835 \\
\hline OASL & 22 & 10 & 22.5 & 0.51637 & 0.5 & 0.97835 \\
\hline IFI27 & 22 & 14 & 22.5 & 0.77598 & 0.5 & 0.9697 \\
\hline EIF2AK2 & 22 & 10 & 22.5 & 0.51637 & 0.5 & 0.97835 \\
\hline LY6E & 21 & 10 & 22 & 0.55833 & 0.5 & 0.97619 \\
\hline
\end{tabular}

powder which is rich in chlorophyll, and other nutrients can effectively alleviate fatigue in chronic patients. The mechanism of chlorophyll's role in relieving fatigue in pSS patients is unclear. It may be related to the nature of the hepatic enzyme inhibitors that increase the concentrations of immunosuppressant like hydroxychloroquine, which has better control of fatigue. And the capacity of scavenging the oxygen radical as an antioxidant may somewhat improve the fatigue of body.

We have identified gene expression profiles in peripheral blood specific to pSS with fatigue characteristics. The analysis of identified DEGs and pathways in this study will 


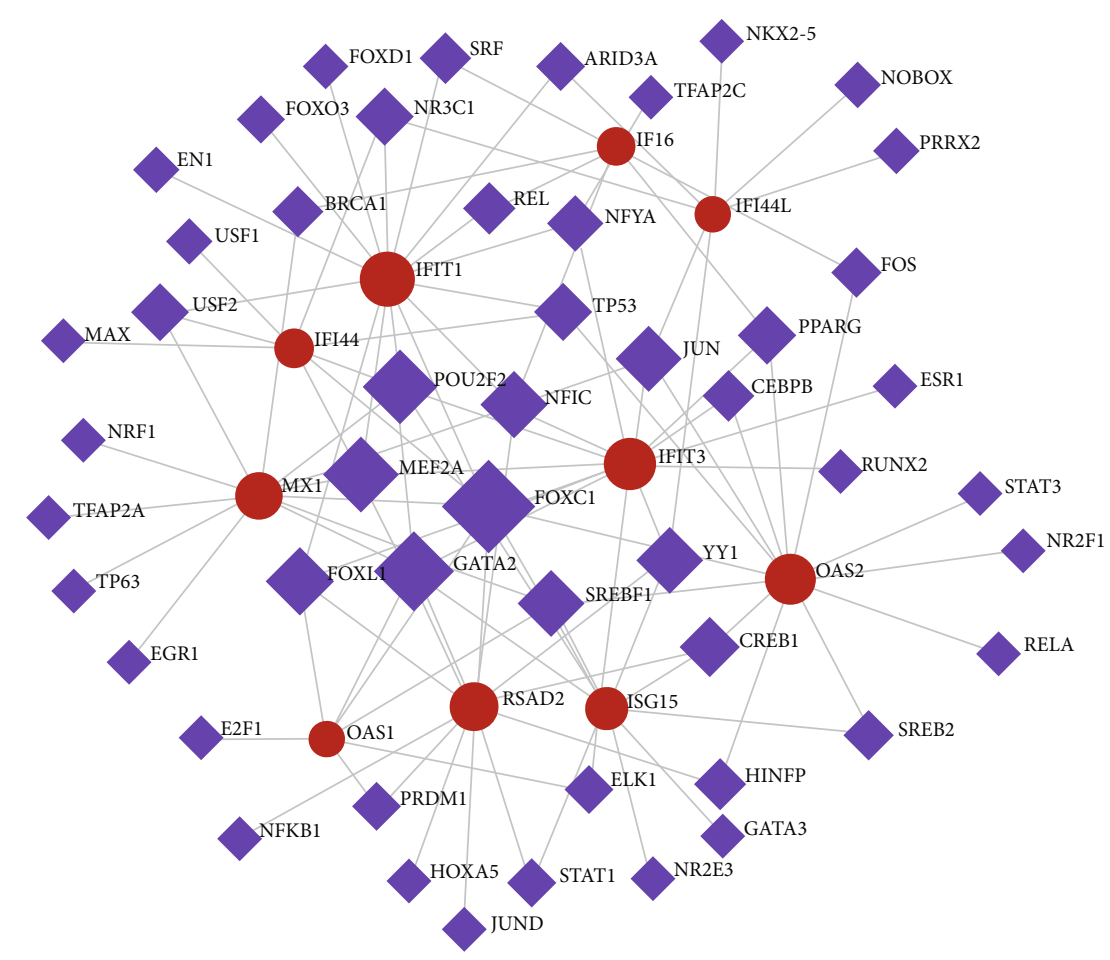

FIGURE 4: Network for TF-gene interaction with common DEGs. The highlighted 10 red color nodes represent the common genes, and other nodes represent TF-genes. The network has 60 nodes and 108 edges.

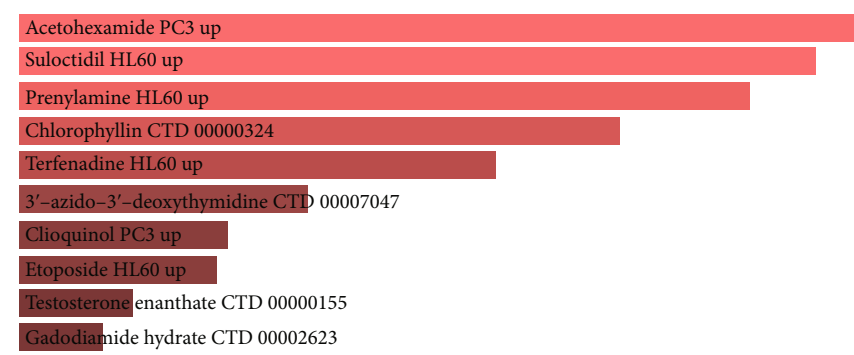

Figure 5: Acetohexamide PC3 UP, suloctidil HL60 UP, prenylamine HL 60 UP, and chlorophyllin CTD 00000324 are the four most polygenic drug molecules that connect with the top 10 hub genes.

TABLE 4: Candidate drug for pSS with fatigue.

\begin{tabular}{|c|c|c|c|}
\hline Name of drugs & $P$ value & $\begin{array}{c}\text { Adjusted } P \\
\text { value }\end{array}$ & Target genes \\
\hline Acetohexamide PC3 UP & $3.47 e-23$ & $8.16 e-21$ & RSAD2; OAS1; OAS2; MX1; IFI6; IFI44; IFIT1; IFI44L \\
\hline Suloctidil HL60 UP & $2.19 e-22$ & $2.57 e-20$ & $\begin{array}{l}\text { RSAD2; OAS1; OAS2; MX1; IFI6; IFI44; ISG15; IFIT1; IFI44L; } \\
\text { IFIT3 }\end{array}$ \\
\hline Prenylamine HL60 UP & $3.46 e-21$ & $2.71 e-19$ & OAS1; MX1; IFI6; IFI44; ISG15; IFIT1; IFI44L; IFIT3 \\
\hline Chlorophyllin CTD 00000324 & $9.59 e-19$ & $5.64 e-17$ & OAS1 OAS2 MX1 IFI6 ISG15 IFIT1 IFIT3 \\
\hline Terfenadine HL60 UP & $1.81 e-16$ & $8.52 e-15$ & OAS1 MX1 IFI6 IFI44 ISG15 IFIT1 IFIT3 \\
\hline $\begin{array}{l}3^{\prime} \text {-Azido-3' -deoxythymidine CTD } \\
00007047\end{array}$ & $6.05 e-13$ & $2.37 e-11$ & RSAD2 OAS1 OAS2 MX1 IFI6 ISG15 IFIT1 IFI44L \\
\hline Clioquinol PC3 UP & $1.79 e-11$ & $6.00 e-10$ & OAS1 MX1 IFI44 IFIT1 IFIT3 \\
\hline Etoposide HL60 UP & $2.92 e-11$ & $8.56 e-10$ & OAS1 MX1 IFI6 IFI44 ISG15 IFIT1 \\
\hline Testosterone enanthate CTD 00000155 & $1.03 e-09$ & $2.68 e-08$ & RSAD2 OAS2 MX1 IFI6 ISG15 IFI44L IFIT3 \\
\hline Gadodiamide hydrate CTD 00002623 & $3.85 e-09$ & $9.05 e-08$ & RSAD2 IFIT1 IFI44L IFIT3 \\
\hline
\end{tabular}


deepen our understanding of the essence of fatigue in pSS. The discovery that chlorophyllin may improve fatigue symptoms provides a theoretical basis for better improving the quality of life in pSS patients. And a preprint has previously been published [56].

\section{Data Availability}

The dataset supporting the conclusions of this article is available in the UK registry of primary Sjögren's syndrome repository and in the hyperlink (https://www.ncbi.nlm.nih .gov/geo/geo2r/?acc=GSE66795).

\section{Ethical Approval}

GEO belongs to public databases. The patients we choose involved in the database have obtained ethical approval. It is available for all users to download relevant data for free. Our study is based on open-source data, so there is no need to offer ethics approval.

\section{Consent}

There is no need for consent to participate.

\section{Conflicts of Interest}

The authors declare that they have no competing interests.

\section{Authors' Contributions}

Guangshu Chen and Li Che contributed equally to this work.

\section{Acknowledgments}

We sincerely acknowledge the GEO database for providing their platforms and contributors for uploading their meaningful datasets. This study was financially supported by the Guangdong Science and Technology Project Fund for Key Scientific Research Base under Grant no. 2019B020230001.

\section{References}

[1] R. I. Fox, F. V. Howell, R. C. Bone, and P. E. Michelson, "Primary Sjogren syndrome: clinical and immunopathologic features," Seminars in Arthritis and Rheumatism, vol. 14, no. 2, pp. 77-105, 1984.

[2] S. E. Gabriel and K. Michaud, "Epidemiological studies in incidence, prevalence, mortality, and comorbidity of the rheumatic diseases," Arthritis Research \& Therapy, vol. 11, no. 3, p. 229, 2009.

[3] K. Asmussen, V. Andersen, G. Bendixen, M. Schiodt, and P. Oxholm, "A new model for classification of disease manifestations in primary Sjögren's syndrome: evaluation in a retrospective long-term study," Journal of Internal Medicine, vol. 239, no. 6, pp. 475-482, 1996.

[4] K. Haldorsen, I. Bjelland, A. I. Bolstad, R. Jonsson, and J. Brun, "A five-year prospective study of fatigue in primary Sjögren's syndrome," Arthritis Research \& Therapy, vol. 13, no. 5, p. R167, 2011.
[5] T. Karageorgas, S. Fragioudaki, A. Nezos, D. Karaiskos, H. M. Moutsopoulos, and C. P. Mavragani, "Fatigue in primary Sjögren's syndrome: clinical, laboratory, psychometric, and biologic associations," Arthritis Care \& Research, vol. 68, no. 1, pp. 123-131, 2016.

[6] C. L. Overman, M. B. Kool, J. A. P. da Silva, and R. Geenen, "The prevalence of severe fatigue in rheumatic diseases: an international study," Clinical Rheumatology, vol. 35, no. 2, pp. 409-415, 2016.

[7] A. Lerdal, A. Wahl, T. Rustoen, B. R. Hanestad, and T. Moum, "Fatigue in the general population: a translation and test of the psychometric properties of the Norwegian version of the fatigue severity scale," Scandinavian Journal of Public Health, vol. 33, no. 2, pp. 123-130, 2005.

[8] N. Howard Tripp, J. Tarn, A. Natasari et al., "Fatigue in primary Sjögren's syndrome is associated with lower levels of proinflammatory cytokines," RMD Open, vol. 2, no. 2, article e000282, 2016.

[9] K. Brække Norheim, J. Imgenberg-Kreuz, K. Jonsdottir et al., "Epigenome-wide DNA methylation patterns associated with fatigue in primary Sjögren's syndrome," Rheumatology, vol. 55, no. 6, pp. 1074-1082, 2016.

[10] K. Bårdsen, M. M. Nilsen, J. T. Kvaløy, K. B. Norheim, G. Jonsson, and R. Omdal, "Heat shock proteins and chronic fatigue in primary Sjögren's syndrome," Innate Immunity, vol. 22, no. 3, pp. 162-167, 2016.

[11] R. Dantzer, C. J. Heijnen, A. Kavelaars, S. Laye, and L. Capuron, "The neuroimmune basis of fatigue," Trends in Neurosciences, vol. 37, no. 1, pp. 39-46, 2014.

[12] A. Sturn, J. Quackenbush, and Z. Trajanoski, "Genesis: cluster analysis of microarray data," Bioinformatics, vol. 18, no. 1, pp. 207-208, 2002.

[13] M. L. Lee, F. C. Kuo, G. A. Whitmore, and J. Sklar, "Importance of replication in microarray gene expression studies: statistical methods and evidence from repetitive cDNA hybridizations," Proceedings of the National Academy of Sciences of the United States of America, vol. 97, no. 18, pp. 9834-9839, 2000.

[14] L. Zhang, P. Xu, X. Wang et al., "Identification of differentially expressed genes in primary Sjögren's syndrome," Journal of Cellular Biochemistry, vol. 120, no. 10, pp. 17368-17377, 2019.

[15] G. G. Song, J. H. Kim, Y. H. Seo, S. J. Choi, J. D. Ji, and Y. H. Lee, "Meta-analysis of differentially expressed genes in primary Sjogren's syndrome by using microarray," Human Immunology, vol. 75, no. 1, pp. 98-104, 2014.

[16] E. Clough and T. Barrett, "The Gene Expression Omnibus database," Methods in Molecular Biology, vol. 1418, pp. 93$110,2016$.

[17] A. Subramanian, P. Tamayo, V. K. Mootha et al., "Gene set enrichment analysis: a knowledge-based approach for interpreting genome-wide expression profiles," Proceedings of the National Academy of Sciences of the United States of America, vol. 102, no. 43, pp. 15545-15550, 2005.

[18] A. Doms and M. Schroeder, "GoPubMed: exploring PubMed with the Gene Ontology," Nucleic Acids Research, vol. 33, no. Web Server, pp. W783-W786, 2005.

[19] M. Kanehisa and S. Goto, "KEGG: Kyoto Encyclopedia of Genes and Genomes," Nucleic Acids Research, vol. 28, no. 1, pp. 27-30, 2000.

[20] M. V. Kuleshov, M. R. Jones, A. D. Rouillard et al., "Enrichr: a comprehensive gene set enrichment analysis web server 2016 
update," Nucleic Acids Research, vol. 44, no. W1, pp. W90W97, 2016.

[21] A. Ben-Hur and W. S. Noble, "Kernel methods for predicting protein-protein interactions," Bioinformatics, vol. 21, Suppl 1, pp. i38-i46, 2005.

[22] Z. Ye, F. Wang, F. Yan et al., "Bioinformatic identification of candidate biomarkers and related transcription factors in nasopharyngeal carcinoma," World Journal of Surgical Oncology, vol. 17, no. 1, p. 60, 2019.

[23] G. Zhou, O. Soufan, J. Ewald, R. E. W. Hancock, N. Basu, and J. Xia, "NetworkAnalyst 3.0: a visual analytics platform for comprehensive gene expression profiling and meta-analysis," Nucleic Acids Research, vol. 47, no. W1, pp. W234-W241, 2019.

[24] E. Y. Chen, C. M. Tan, Y. Kou et al., "Enrichr: interactive and collaborative HTML5 gene list enrichment analysis tool," BMC Bioinformatics, vol. 14, no. 1, p. 128, 2013.

[25] L. B. Krupp and D. A. Pollina, "Mechanisms and management of fatigue in progressive neurological disorders," Current Opinion in Neurology, vol. 9, no. 6, pp. 456-460, 1996.

[26] A. M. Mengshoel, K. B. Norheim, and R. Omdal, "Primary Sjögren's syndrome: fatigue is an ever-present, fluctuating, and uncontrollable lack of energy," Arthritis Care \& Research, vol. 66, no. 8, pp. 1227-1232, 2014.

[27] L. C. Pollard, E. H. Choy, J. Gonzalez, B. Khoshaba, and D. L. Scott, "Fatigue in rheumatoid arthritis reflects pain, not disease activity," Rheumatology, vol. 45, no. 7, pp. 885-889, 2006.

[28] C. A. Dinarello, A. Simon, and J. W. van der Meer, “Treating inflammation by blocking interleukin-1 in a broad spectrum of diseases," Nature Reviews. Drug Discovery, vol. 11, no. 8, pp. 633-652, 2012.

[29] B. L. Hart, "Biological basis of the behavior of sick animals," Neuroscience and Biobehavioral Reviews, vol. 12, no. 2, pp. 123-137, 1988.

[30] S. Rossi, V. Studer, C. Motta et al., "Neuroinflammation drives anxiety and depression in relapsing-remitting multiple sclerosis," Neurology, vol. 89, no. 13, pp. 1338-1347, 2017.

[31] J. M. Kim, H. J. Kang, J. W. Kim et al., "Associations of tumor necrosis factor- $\alpha$ and interleukin- $1 \beta$ levels and polymorphisms with post-stroke depression," The American Journal of Geriatric Psychiatry, vol. 25, no. 12, pp. 1300-1308, 2017.

[32] R. Omdal and R. Gunnarsson, "The effect of interleukin-1 blockade on fatigue in rheumatoid arthritis-a pilot study," Rheumatology International, vol. 25, no. 6, pp. 481-484, 2005.

[33] C. Cavelti-Weder, R. Furrer, C. Keller et al., "Inhibition of IL1beta improves fatigue in type 2 diabetes," Diabetes Care, vol. 34, no. 10, article e158, 2011.

[34] S. E. Carsons, F. B. Vivino, A. Parke et al., "Treatment guidelines for rheumatologic manifestations of Sjögren's syndrome: use of biologic agents, management of fatigue, and inflammatory musculoskeletal pain," Arthritis Care \& Research, vol. 69, no. 4, pp. 517-527, 2017.

[35] H. Li, T. R. Reksten, J. A. Ice et al., "Identification of a Sjögren's syndrome susceptibility locus at OAS1 that influences isoform switching, protein expression, and responsiveness to type I interferons," PLoS Genetics, vol. 13, no. 6, article e1006820, 2017.

[36] B. Segal, "Fatigue in primary Sjogren's syndrome," in Sjogren's Syndrome: Diagnosis and Therapeutics, M. Ramos-Casals, Ed., pp. 129-143, Springer Verlag, London, 2012.
[37] B. E. Strombeck, E. Theander, and L. T. Jacobsson, "Effects of exercise on aerobic capacity and fatigue in women with primary Sjogren's syndrome," Rheumatology, vol. 46, no. 5, pp. 868-871, 2007.

[38] V. Sankar, M. T. Brennan, M. R. Kok et al., "Etanercept in Sjögren's syndrome: a twelve-week randomized, double-blind, placebo-controlled pilot clinical trial," Arthritis and Rheumatism, vol. 50, no. 7, pp. 2240-2245, 2004.

[39] X. Mariette, P. Ravaud, S. Steinfeld et al., "Inefficacy of infliximab in primary Sjögren's syndrome: results of the randomized, controlled Trial of Remicade in Primary Sjögren's Syndrome (TRIPSS)," Arthritis and Rheumatism, vol. 50, no. 4, pp. 1270-1276, 2004.

[40] S. Suryavanshi, D. Sharma, R. Checker et al., "Amelioration of radiation-induced hematopoietic syndrome by an antioxidant chlorophyllin through increased stem cell activity and modulation of hematopoiesis," Free Radical Biology \& Medicine, vol. 85, pp. 56-70, 2015.

[41] K. K. Boloor, J. P. Kamat, and T. P. Devasagayam, "Chlorophyllin as a protector of mitochondrial membranes against $\gamma$-radiation and photosensitization," Toxicology, vol. 155, no. 1-3, pp. 63-71, 2000.

[42] C. H. Yun, H. G. Jeong, J. W. Jhoun, and F. P. Guengerich, "Non-specific inhibition of cytochrome P450 activities by chlorophyllin in human and rat liver microsomes," Carcinogenesis, vol. 16, no. 6, pp. 1437-1440, 1995.

[43] L. C. Chiu, C. K. Kong, and V. E. Ooi, "The chlorophyllininduced cell cycle arrest and apoptosis in human breast cancer MCF-7 cells is associated with ERK deactivation and cyclin D1 depletion," International Journal of Molecular Medicine, vol. 16, no. 4, pp. 735-740, 2005.

[44] J. W. Fahey, K. K. Stephenson, A. T. Dinkova-Kostova, P. A. Egner, T. W. Kensler, and P. Talalay, "Chlorophyll, chlorophyllin and related tetrapyrroles are significant inducers of mammalian phase 2 cytoprotective genes," Carcinogenesis, vol. 26, no. 7, pp. 1247-1255, 2005.

[45] J. E. Mata, Z. Yu, J. E. Gray, D. E. Williams, and R. RodriguezProteau, "Effects of chlorophyllin on transport of dibenzo( $a$, l)pyrene, 2-amino-1-methyl-6-phenylimidazo-[4,5- $b$ ]pyridine, and aflatoxin $\mathrm{B}_{1}$ across Caco- 2 cell monolayers," Toxicology, vol. 196, no. 1-2, pp. 117-125, 2004.

[46] Coronavirus Resource Center, Johns Hopkins University, “COVID-19 Dashboard," November 2021, https:// coronavirus.jhu.edu/map.html.

[47] E. Terpos, I. Ntanasis-Stathopoulos, I. Elalamy et al., "Hematological findings and complications of COVID-19," American Journal of Hematology, vol. 95, no. 7, pp. 834-847, 2020.

[48] Q. Zhao, M. Meng, R. Kumar et al., "Lymphopenia is associated with severe coronavirus disease 2019 (COVID-19) infections: a systemic review and meta-analysis," International Journal of Infectious Diseases, vol. 96, pp. 131-135, 2020.

[49] M. Zheng, Y. Gao, G. Wang et al., "Functional exhaustion of antiviral lymphocytes in COVID-19 patients," Cellular \& Molecular Immunology, vol. 17, no. 5, pp. 533-535, 2020.

[50] N. F. Clark and A. W. Taylor-Robinson, "COVID-19 therapy: could a copper derivative of chlorophyll a be used to treat lymphopenia associated with severe symptoms of SARS-CoV-2 infection?," Frontiers in Medicine, vol. 8, article 620175, 2021.

[51] C. Ulbricht, R. Bramwell, M. Catapang et al., "An evidencebased systematic review of chlorophyll by the Natural 
Standard Research Collaboration," Journal of Dietary Supplements, vol. 11, no. 2, pp. 198-239, 2014.

[52] B. Mysliwa-Kurdziel and K. Solymosi, "Phycobilins and phycobiliproteins used in food industry and medicine," Mini Reviews in Medicinal Chemistry, vol. 17, no. 13, pp. 11731193, 2017.

[53] M. G. Ferruzzi, M. L. Failla, and S. J. Schwartz, "Sodium copper chlorophyllin: in vitro digestive stability and accumulation by Caco-2 human intestinal cells," Journal of Agricultural and Food Chemistry, vol. 50, no. 7, pp. 2173-2179, 2002.

[54] S. Bhatia, P. N. Prabhu, A. C. Benefiel et al., "Galacto-oligosaccharides may directly enhance intestinal barrier function through the modulation of goblet cells," Molecular Nutrition \& Food Research, vol. 59, no. 3, pp. 566-573, 2015.

[55] Y. Zeng, X. Pu, J. Yang et al., "Preventive and therapeutic role of functional ingredients of barley grass for chronic diseases in human beings," Oxidative Medicine and Cellular Longevity, vol. 2018, Article ID 3232080, 15 pages, 2018.

[56] G. Chen, L. Che, X. Cai, P. Zhu, J. Ran, and S. Liu, Bioinformatic analysis identifies biomarkers and treatment targets in primary Sjögren's syndrome patients with fatigue, Research Square, 2021. 